\title{
GW23-e2272 SICK SINUS SYNDROME RELATED WITH GASTRIC CANCER: ONE CASE REPORT
}

doi:10.1136/heartjnl-2012-302920n.14

Xie Dong-yang, Zhong Yi-ming. Department of Cardiology, Affiliated Hospital of Gannan Medical College

Objectives We report a 58-year-old male who was admitted due to gastric antrum adenocarcinoma diagnosed by gastroscopy and pathology. He had no heart disease and syncope in his past medical history. Cardiac structure and function is also normal confirmed by echocardiography. 24-h ambulatory ECG examinations showed the average heart rate was 51 beats/min, the fastest heart rate was 62 beats/min, and the slowest was 35 beats/min. Atropine test was positive (the fastest heart rate was 71 beats $/ \mathrm{min}$ )

Methods Therefore, the patient was diagnosed as sick sinus syndrome and temporary cardiac pacing was administrated during the perioperative to ensure the safety. After surgery of gastric cancer D2 radical resection, we re-examine the 24-h ambulatory ECG, which showed that it was sinus rhythm, mean heart rate was 69 beats/min, the fastest heart rate was 122 beats/min, and the slowest was 55 beats/min.

Results Then the temporary pacemaker was removed. The routine ECG showed that he had sinus rhythm and heart rate was 6785 beats/min in 1 month and 3 month follow-up. Auscultation of heart rate after activity was about 130 beats/min. In this case report, patients with gastric cancer had significant bradycardia before surgery and returned to normal after surgery. Gastric cancer may be stimulated vagus nerve and cause bradycardia.

Conclusions Upon inquiry, there is no report found that the correlation of gastric cancer and bradycardia and it is easy to be misdiagnosis. We analysis that there is a lot of the vagus nerve in the abdominal organs, and organ lesions can lead to stimulation of vagus nerve. The spinal nerve T4-6 common dominates the heart, the stomach and duodenum. Abdominal organ disease accompanies with sinus bradycardia should be suspected vagus nerve reflex. If condition may be given temporary pacing to pass the critical stage, even if they have symptoms of bradycardia, permanent pacing should be suspended implanted in order to avoid unnecessary waste of medical resources, to bring patients with additional injury, the risk of accidents, and psychological and economic burden. 